\title{
NOVA OUTBURSTS DUE TO ACCRETION FROM ROTATING H-RICH DISKS ONTO WHITE DWARFS
}

\author{
Warren M. Sparks
}

and

\section{G. Siegfried Kutter*}

NASA/Goddard Space Flight Center

Laboratory for Astronomy and Solar Physics

Greenbelt, Maryland 20771

In this paper we discuss the third stage of our research on the nova outburst as described in the preceeding paper (Kutter and Sparks, page 290), i.e. we accrete hydrogen-rich material (normal solar composition) with Keplerian velocity onto a helium white dwarf at a rate of $10^{-8} \mathrm{M} / \mathrm{yr}$. When material with high angular momentum from a circumstellar disk is accreted onto a white dwarf with negligible angular momentum, a tremendous shear instability is created, and hydrogen-rich material is mixed with helium-rich material of the white dwarf on a scale that is short compared to the accretion time scale. Following Kippenhahn and Thomas (1978), we assume that in the mixing region marginal stability is established. Mathematically this is expressed by setting the Richardson number (the ratio of the work done against buoyancy to the kinetic energy of the turbulence) equal to $\frac{1}{4}$.

The results are shown in Figure 1 which is a plot of the accreted hydrogen rich mass fraction as a function of the mass inward from the surface at various times. The remaining mass fraction is the original white dwarf material, which we assume to be the normal solar composition with all of the hydrogen converted into helium. As the evolution proceeds, the accreting hydrogen-rich material penetrates deeper into the star and its frational abundance increases. Because of this penetration we find that (1) the thermonuclear runaway occurs in deeper layers where the e-degeneracy is higher and (2) the shear turbulence mixes to a depth of about 10 times the mass of the accreted material where it might reach the carbon/oxygen core of the white dwarf. Both of these effects strengthen the thermonuclear runaway, the first by delaying the expansion of the star in response to the energy released by the thermonuclear reactions and the second by supplying CNO nuclei. We believe that both of these effects are necessary conditions for a fast nova outburst and that together they are a sufficient condition.

* On leave of absence from The Evergreen State College, Olympia, WA 


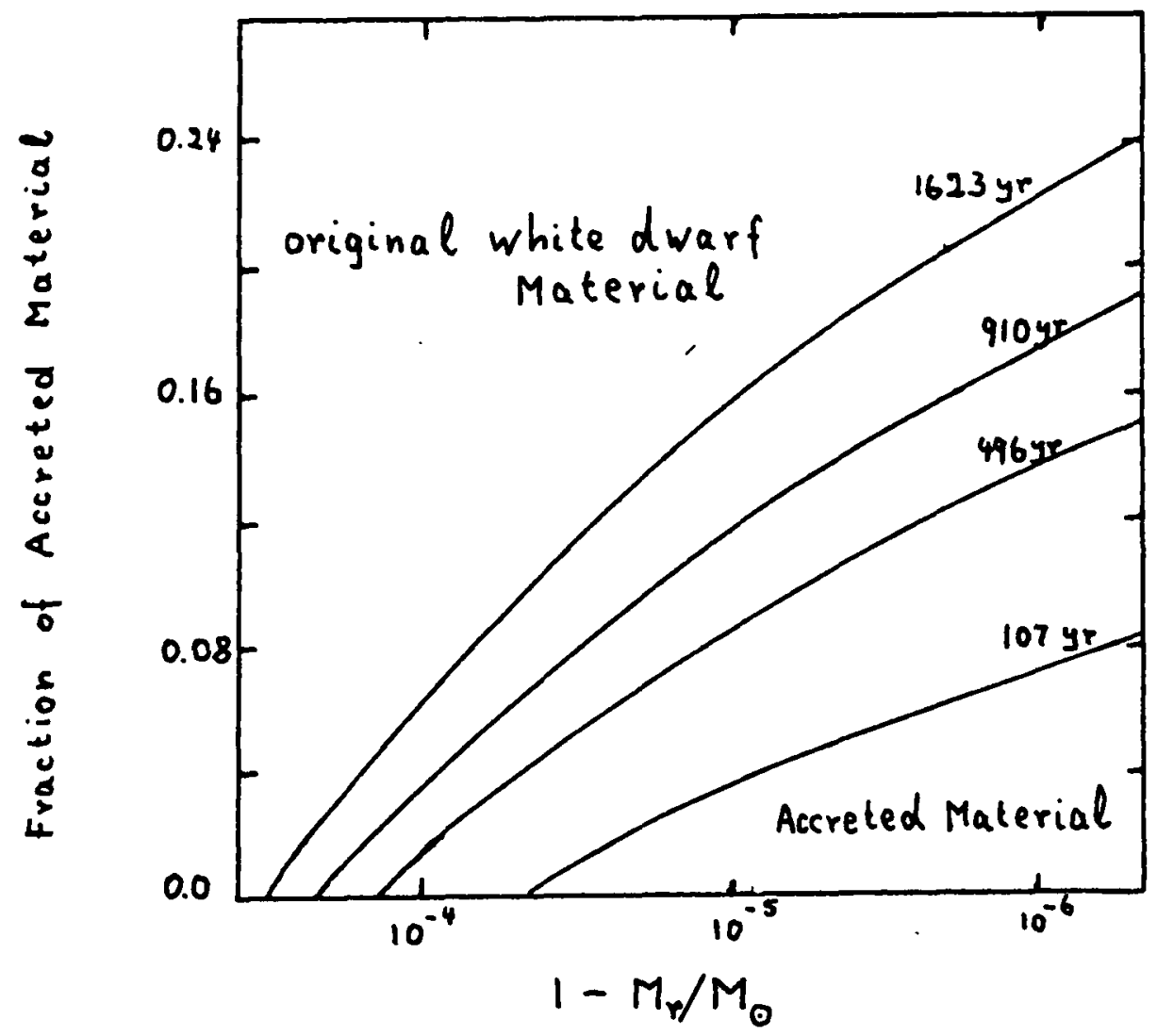

Fig. I

While carrying out the numerical work of this evolution we discovered an unusual mechanism of energy transport in the mixing region. Let us consider the schematic diagram of temperature versus radius in Fig. 2. In white dwarf models the adiabatic temperature gradient is steeper than the actual temperature gradient. An element of material that is forcibly mixed inward by the shear instability finds itself at a higher temperature than its sourroundings, and it contracts by giving up energy. At the same time an equivalent element of material, mixed outward, is cooler than its environment, and it expands and heats. When this shear mixing sequence is plotted in the $\mathrm{P}-\mathrm{V}$ plane shown in Fig. 3, the cycle moves in a counterclockwise direction, i.e., it is a refrigeration cycle! The net result is that energy is transported from the cooler outside layers to the hotter deeper layers. The energy flow is inward and against the temperature gradient. Another way to view this process is by considering Fig. 2 . As with convection, the process of mixing drives the temperature gradient toward the adiabat. However, in the case of shear mixing the approach is from below toward the adiabatic gradient, which is Just the opposite of convection. In the process of steepening the temperature gradient, energy flows inward. 


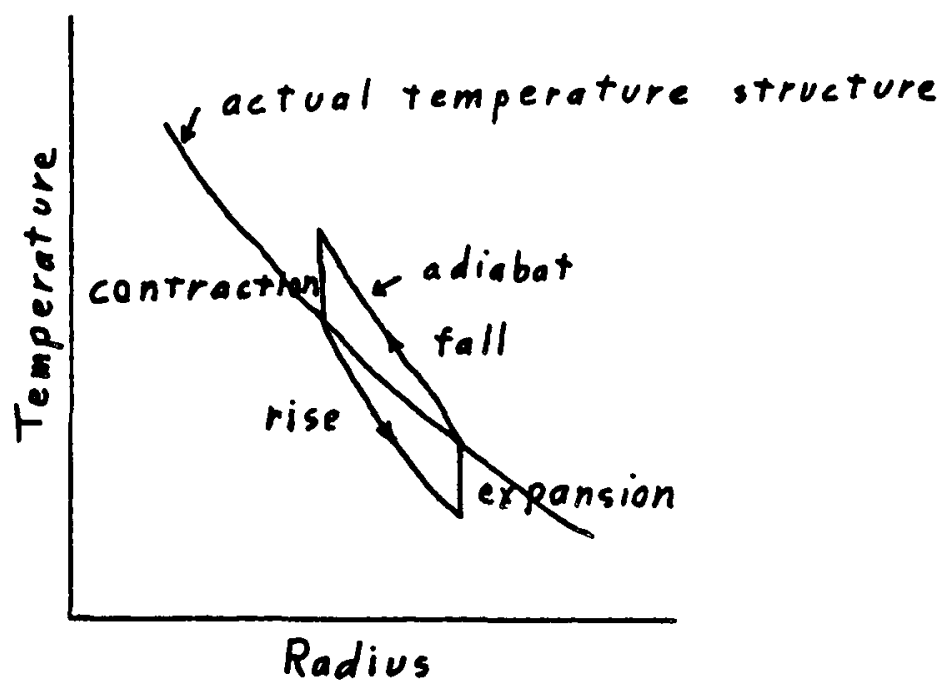

Fig. 2

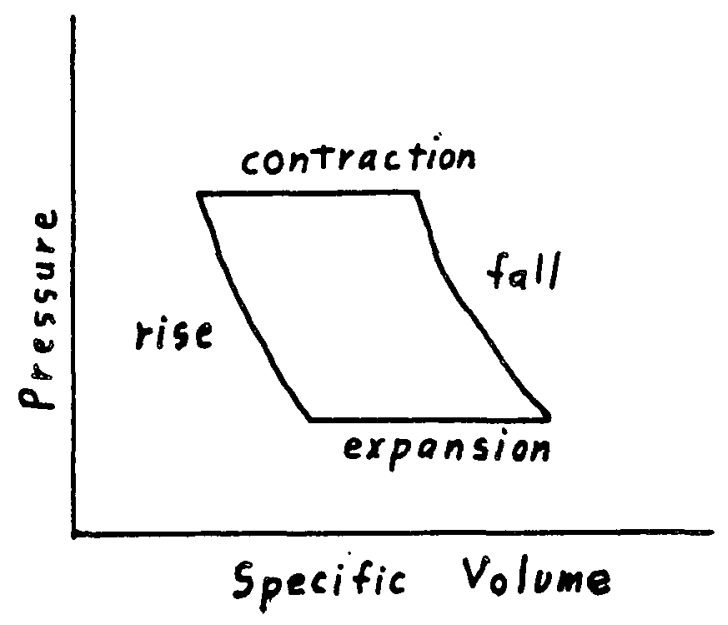

Fig. 3

We are continuing our study of the accretion of material with angular momentum, by including ( 1 ) the $\mathrm{P}-\mathrm{V}$ work term in the Richardson number and (2) the energy transportation by shear mixing. In summary we find that the angular momentum of the accreted material determines the temperature and composition stmucture of the white dwarf's envelope leading up to the thermonuclear runaway.

\section{References}

Kippenhahn, R., and Thomas, H.-C. 1978, Astr. Ap., 63, 265. 